\title{
Health and Oral Health Care Needs and Health Care-Seeking Behavior Among Homeless Injection Drug Users in San Francisco
}

Jonathan Leserman Robbins, Lynn Wenger, Jennifer Lorvick, Caroline Shiboski, and Alex H. Kral

\begin{abstract}
Few existing studies have examined health and oral health needs and treatment-seeking behavior among the homeless and injection drug users (IDUs). This paper describes the prevalence and correlates of health and oral health care needs and treatment-seeking behaviors in homeless IDUs recruited in San Francisco, California, from 2003 to 2005 (N=340). We examined sociodemographic characteristics, drug use patterns, HIV status via oral fluid testing, physical health using the Short Form 12 Physical Component Score, self-reported needs for physical and oral health care, and the self-reported frequency of seeking medical and oral health care. The sample had a lower health status as compared to the general population and reported a frequent need for physical and oral health care. In bivariate analysis, being in methadone treatment was associated with care-seeking behavior. In addition, being enrolled in Medi-Cal, California's state Medicaid program, was associated with greater odds of seeking physical and oral health care. Methamphetamine use was not associated with higher odds of needing oral health care as compared to people who reported using other illicit drugs. Homeless IDUs in San Francisco have a large burden of unmet bealth and oral health needs. Recent cuts in Medi-Cal's adult dental coverage may result in a greater burden of oral health care which will need to be provided by emergency departments and neighborhood dental clinics.
\end{abstract}

KEYwordS IDU, Homeless, Health Care, Oral Health, Methamphetamine, Dental Care

\section{INTRODUCTION}

Quotidian aspects of injection drug use-such as oral health and health care access-remain understudied, ${ }^{1}$ while researchers have tended to study catastrophic and life-threatening conditions, including human immunodeficiency virus (HIV), hepatitis B virus, hepatitis $C$ virus, tuberculosis, and accidental overdose. ${ }^{2-5}$ Recent international research has provided evidence that dental problems are common among injection drug users (IDUs), ${ }^{6-9}$ but few researchers have explored the oral health of IDUs within the United States.

Robbins is with the Joint Medical Program, University of California, Berkeley and University of California, San Francisco, Berkeley, CA, USA; Wenger, Lorvick, and Kral are with the University of California, San Francisco, RTI International, San Francisco, CA, USA; Shiboski is with the Department of Orofacial Sciences, School of Dentistry, University of California, San Francisco, CA, USA; Lorvick is with the School of Public Health, University of California, Berkeley, Berkeley, CA, USA; Kral is with the Department of Family and Community Medicine, University of California, San Francisco, CA, USA.

Correspondence: Jonathan Leserman Robbins, Joint Medical Program, University of California, Berkeley and University of California, San Francisco, 570 University Hall, Berkeley, CA 94720-1190, USA. (E-mail: jonathan.robbins@ucsf.edu) 
In the 1970s, heroin use was found to be associated with increased numbers of decayed, missing, and filled teeth. ${ }^{10,11}$ Long-term heroin addiction was thought to be related to diminished oral health status either through direct physiologic effects, or more likely, through poor personal hygiene and chronic malnutrition. ${ }^{10}$ More recently, injection and non-injection methamphetamine use has been hypothesized to cause dental caries likely due to reduced salivation. ${ }^{12-14}$ In a well-designed study of oral health and methamphetamine, a cohort of methamphetamine users was found to have significantly more missing teeth and a higher likelihood of reporting oral health problems than a matched comparison group of non-methamphetamine users from a national health survey. ${ }^{15}$ Intravenous use of methamphetamine was significantly more likely to be associated with missing teeth than smoking the drug. ${ }^{15}$ Subsets of methamphetamine users in the study also expressed concerns about dental appearance, problems with broken or loose teeth, persistent tooth grinding, and erosion. ${ }^{15}$

Although drug injection has been independently associated with having untreated oral health problems, ${ }^{16}$ the proportion of IDUs seeking oral health services is low. ${ }^{17}$ Despite the high prevalence of chronic and acute illness associated with injection drug use, access to preventive care among IDUs remains low. ${ }^{18-21}$ IDUs have also been found to have fewer outpatient medical visits than non-drug users, and health care costs for drug users exceed that of non-drug users by 1,000 USD per year. ${ }^{22}$ Many researchers have called for expanded access to outpatient ambulatory care, primary health care screenings, and routine immunization for IDUs. ${ }^{19,21-23}$

The purpose of this paper is to better describe the prevalence and correlates of health care needs and care-seeking behavior of homeless IDUs in San Francisco, as they apply to both physical and oral health. By learning more about the scope of unmet need for preventive medical and oral health care for homeless IDUs, we will provide data that can help guide health care policy as it pertains to the urban poor.

\section{METHODS}

\section{Sampling Design}

As part of a 6-month prospective cohort study of homeless IDUs funded by the United States' National Institute of Drug Abuse, study participants were recruited between April 2003 and July 2005 from three neighborhoods in San Francisco: the Mission District, South of Market (SoMa), and the Tenderloin. The Mission cohort was a convenience sample of IDUs recruited from a newly opened integrated resource center for the homeless and marginally housed called the Mission Neighborhood Resource Center. Participants from the SoMa and Tenderloin districts were recruited using targeted sampling methods. ${ }^{24,25}$

Study eligibility was limited to clients who were active IDUs (past 30 days), as verified by visible signs of recent injection drug use ("tracks" or recently punctured veins), and at least 18 years of age. Those participants meeting all eligibility criteria were referred to the Project Director who explained the study procedures and provided an appointment for interview the same day. Interviews lasting 30-45 min were administered in a private setting by trained interviewer/counselors using a Computer-Assisted Personal Interview, which was programmed using the Questionnaire Development System (QDS, NOVA Research, Bethesda, MD, USA). All participants gave informed consent to participate in the study and signed Health 
Insurance Portability and Accountability consent forms to give project staff access to the participant's health system utilization records prior to the interview. After the interview, respondents were advised about their risk behaviors, given HIV-1 pretest counseling, and referred to medical and social services as needed. Participants provided either an oral fluid sample for OraSure HIV-1 antibody testing or a blood sample for HIV-1 antibody testing. Participants were paid 15 USD to compensate them for their time spent during the interview and were asked to return 2 weeks post-interview for HIV test results and counseling.

\section{Variables}

The study survey included information about demographics, health and mental health status, physical and oral health services care-seeking behavior, drug use, and drug treatment utilization. Four outcome variables were identified for the current analysis, all of which were self-reported with a 6-month time frame: needing health care at least six times in the 6 months prior to the study (average of monthly), seeking health care, needing dental care at least six times in the 6 months prior to the study (average of monthly), and seeking dental care. The four outcome variables were operationalized as binary outcomes (yes/no). The needing health and oral health care variables were operationalized as binary outcomes to facilitate interpretation. The choice of needing care an average of monthly was a more appropriate outcome variable for this underserved population, as almost all participants reported needing care at least once in a 6-month time frame.

Independent variables in the study included gender, ethnicity, age, high school graduation or equivalent, California state Medicaid coverage (Medi-Cal), not having medical or dental insurance, being in methadone maintenance or detoxification treatment, HIV serostatus via OraSure HIV-1 antibody testing, number of years injecting drugs, and use of injected or non-injected substances including heroin, speedballs (an injected combination of heroin and cocaine), cocaine, crack cocaine, methamphetamine, prescription opiates, and benzodiazepines, as well as alcohol and marijuana. Health status was assessed using the Physical Component Score (PCS) of the Short Form 12 (SF-12) health survey, a standardized measure of the severity of health symptoms that has been shown to be a reliable and valid measure in marginally housed and homeless populations. ${ }^{26,27}$ The SF-12 consists of 12 closedended questions which are then standardized to a composite score. The SF-12 PCS was created to approach a mean of 50 with a standard deviation of 10 in a representative sample of the US population, with variation by 1 to 3 points by age deciles. ${ }^{26}$ Higher scores indicate better health.

\section{Analysis}

Prior to analyzing the data, we utilized analysis of variance to assess whether there were any biases in independent and outcome variables attributable to neighborhood. We aggregated the data from neighborhood and sampling technique into one large sample, adjusting for differences in neighborhood and sampling technique effects as appropriate in multivariate models.

Bivariate analyses were conducted to assess association between each independent variable and each of the four outcome variables. All variables that were significantly associated with the outcome measures in bivariate analyses $(p<0.10)$ were entered into forward and backward stepwise non-hierarchical logistic regression models to assess which were independently associated with the outcomes. The resulting models excluded variables that were not statistically associated with 
the outcome $(p>0.05)$. Theoretically plausible interaction effects were tested, and standard regression diagnostics were employed including analysis of residuals, examination of the influence of outliers, multi-collinearity, and scaling adjustments. Data analysis was performed using STATA version 10.0 (College Station, Texas). ${ }^{28}$

\section{RESULTS}

Overall, we conducted 398 interviews in three neighborhoods from 2003 to 2005. We excluded individuals who did not answer yes to the question "Do you consider yourself homeless?" during the interview from analysis $(n=58)$ in order to reflect the project's emphasis on homeless IDU and to account for a potential immeasurable confounder attributable to sampling technique. The excluded participants $(n=58)$ of self-reported non-homeless had a higher percentage of Medi-Cal coverage versus the homeless $(n=340)$ participants $(32.8 \%$ homeless, $21.0 \%$ non-homeless; $p<0.05)$. Otherwise, there were no statistically significant differences. The present paper reports on our baseline cross-sectional data of 340 homeless IDUs [Mission $(n=$ 154), SoMa $(n=110)$, and Tenderloin $(n=76)]$.

\section{Sample Characteristics}

Nearly one-quarter of the sample was women, nearly one-half was white, and the mean age was 42.8 years (Table 1 ). Twelve percent tested positive for HIV antibodies and the mean SF-12 PCS was 43.5. Variation by neighborhood was found for gender, and white or Latino ethnicity $(p<0.05)$. Other demographic variables did not significantly vary across neighborhood. Variation by sampling technique [convenience $(n=154)$ vs. targeted $(n=186)$ ] was found for gender and white or Latino ethnicity.

TABLE 1 Demographic characteristics of homeless injection drug users in San Francisco

\begin{tabular}{ll}
\hline & $\%(N=340)$ \\
\hline Female & 23.2 \\
Ethnicity & 46.8 \\
White & 37.1 \\
African-American & 7.0 \\
Latino & 9.1 \\
Other & 68.8 \\
High school graduate or equivalent & \\
Insurance coverage & 21.0 \\
Medi-Cal coverage & 75.0 \\
No health insurance & \\
Methadone treatment & 18.8 \\
Any methadone use in past 6 months & 9.4 \\
Methadone detoxification in past 6 months & 11.7 \\
Methadone maintenance in past 6 months & 12.1 \\
HIV antibody-seropositive & 21.2 \\
Drinks alcohol every day & Mean (SD) \\
Continuous variables & $42.8(9.0)$ \\
Age in years & $43.5(11.4)$ \\
SF-12 Physical Component Score (PCS) & $22.3(10.9)$ \\
Number of years of injection drug use &
\end{tabular}


Study participants used a wide variety of illicit substances, with $82 \%$ reporting poly-drug use in the past 6 months (Table 2). Injection heroin and smoking crack cocaine were the most commonly used substances. Speedballs, methamphetamine, prescription opiates, and marijuana were used by approximately half the participants.

\section{Health Care Needs and Care-Seeking Behavior}

Medical and oral health care needs were common (Table 3). Of the $82 \%$ of participants who reported a need for medical care in the past 6 months, $72 \%$ sought any care. Among the $63 \%$ of participants who reported a need for oral health care in the past 6 months, only $27 \%$ sought care. Forty percent of participants reported they had needed health care at least six times in the past 6 months, while $31 \%$ of participants reported needing dental care at least six times in the previous 6 months. In 6 months, 199 participants (59\% of the sample) reported seeking medical care at least once for a total of 1,506 times, of which $22 \%$ went to an emergency department, $6 \%$ to the Mission Neighborhood Resource Center, and $72 \%$ went elsewhere. In 6 months, 108 participants (32\% of the sample) reported having sought oral health care at least once for a total of 142 times, of which $8 \%$ went to an emergency department, $30 \%$ to the Mission Neighborhood Resource Center, and $62 \%$ went elsewhere.

\section{Needing Frequent Health Care}

To assess whether participant demographics and substance abuse were associated with needing frequent health care (six or more times in past 6 months), we conducted bivariate and multivariate analyses. In bivariate analysis, needing frequent health care was associated with lower SF-12 PCS (odds ratio $[\mathrm{OR}]=0.93$; $95 \%$ confidence interval $[\mathrm{CI}] 0.91,0.95 ; p<0.001)$ and higher odds of methadone treatment $(\mathrm{OR}=2.12 ; 95 \% \mathrm{CI} 1.23,3.68 ; p=0.007)$, prescription opiate use $(\mathrm{OR}=$ $2.04 ; 95 \%$ CI $1.31,3.18 ; p=0.002)$, and benzodiazepine use $(\mathrm{OR}=2.40 ; 95 \% \mathrm{CI}$ $1.44,4.02 ; p=0.001$ ) (Table 4). A one standardized unit increase in SF-12 PCS (better health) would decrease the odds of needing care by $7 \%$. In multivariate analysis, no variables were significantly associated with needing frequent health care once the SF-12 variable was entered.

\section{Seeking Health Care}

In bivariate analysis, Medi-Cal insurance (OR $=2.11 ; 95 \%$ CI 1.19, 3.74; $p=0.01)$, methadone treatment $(\mathrm{OR}=3.39 ; 95 \%$ CI $1.77,6.52 ; p<0.001)$, and a frequent need for health care $(\mathrm{OR}=3.44 ; 95 \%$ CI 2.13, 5.55; $p<0.001)$ were associated with

TABLE 2 Substance use among homeless injection drug users in San Francisco $(N=340)$

\begin{tabular}{lccc}
\hline & Injected (\%) & Non-injected (\%) & Any use (\%) \\
\hline Heroin & 78.2 & 16.5 & 79.4 \\
Speedball (heroin/cocaine) & 50.0 & 7.3 & 50.0 \\
Cocaine & 25.2 & 13.0 & 31.2 \\
Crack cocaine & 16.0 & 72.1 & 74.0 \\
Methamphetamine & 45.6 & 25.0 & 47.7 \\
Prescription opiates & 10.1 & 42.1 & 44.3 \\
Benzodiazepines & $(\mathrm{n} / \mathrm{a})$ & 23.1 & 23.1 \\
Marijuana & $(\mathrm{n} / \mathrm{a})$ & 54.4 & 54.4 \\
\hline
\end{tabular}


TABLE 3 Medical and oral health care service needs and care-seeking behavior of the preceding 6 months

\begin{tabular}{lc}
\hline & $\%(N=340)$ \\
\hline Reported needing any health care & 81.8 \\
Reported needing health care at least 6 times & 39.7 \\
Sought health care if reported needing health care & 71.6 \\
Sought health care (all participants) & 58.5 \\
Reported needing any oral health care & 63.2 \\
Reported needing oral health care at least 6 times & 30.6 \\
Sought oral health care if reported needing oral health care & 27.0 \\
Sought oral health care (all participants) & 31.8 \\
\hline
\end{tabular}

increased odds of seeking health care. Having no health insurance $(\mathrm{OR}=0.50 ; 95 \%$ CI $0.29,0.85 ; p=0.010)$ and the SF-12 PCS $(\mathrm{OR}=0.95 ; 95 \%$ CI 0.93, 0.97; $p<0.001)$ were associated with decreased odds of seeking health care. Medi-Cal and methadone maintenance were collinear because Medi-Cal is one way of obtaining methadone treatment with few out-of-pocket costs. In the multivariate model, methadone treatment (adjusted odds ratio $[\mathrm{AOR}]=2.29 ; 95 \%$ CI 1.24, 4.24; $p=0.008)$ and needing frequent health care $(\mathrm{AOR}=2.67 ; 95 \%$ CI 1.65, 4.34; $p<0.001$ ) were independently associated with increased odds of seeking care.

\section{Needing Frequent Oral Health Care}

There were no statistically significant positive associations between needing frequent oral health care and the explanatory variables. High school graduation or the equivalent was significantly associated with a decreased reported need for frequent dental care (six times or more in past 6 months) in bivariate analysis $(\mathrm{OR}=0.55$; $95 \%$ CI $0.34,0.89 ; p=0.016)$. In bivariate analysis, any methamphetamine use (either injection or non-injection) was not significantly associated with greater odds of needing frequent dental care than non-users $(\mathrm{OR}=1.28,95 \%$ CI $0.80,2.03$;

TABLE 4 Selected bivariate odds ratios $(p<0.05)$ for the four outcome variables

\begin{tabular}{|c|c|c|c|c|}
\hline Outcome variable & Characteristic & Odds ratio & $95 \% \mathrm{Cl}$ & $p$ value \\
\hline \multirow{4}{*}{$\begin{array}{l}\text { Reported needing health } \\
\text { care at least } 6 \text { times }\end{array}$} & Benzodiazepine use & 2.40 & $1.44,4.02$ & 0.001 \\
\hline & Methadone treatment & 2.12 & $1.23,3.68$ & 0.007 \\
\hline & Prescription opiate use & 2.04 & $1.31,3.18$ & 0.002 \\
\hline & SF-12 PCS & 0.93 & $0.91,0.95$ & $<0.001$ \\
\hline \multirow[t]{5}{*}{ Sought health care } & $\begin{array}{l}\text { Frequent need for } \\
\text { health care }\end{array}$ & 3.44 & $2.13,5.55$ & $<0.001$ \\
\hline & Medi-Cal insurance & 2.11 & $1.19,3.74$ & 0.01 \\
\hline & Methadone treatment & 3.39 & $1.77,6.52$ & $<0.001$ \\
\hline & No health insurance & 0.50 & $0.29,0.85$ & 0.010 \\
\hline & SF-12 PCS & 0.95 & $0.93,0.97$ & $<0.001$ \\
\hline $\begin{array}{l}\text { Reported needing oral health } \\
\text { care at least } 6 \text { times }\end{array}$ & $\begin{array}{l}\text { High school graduation } \\
\text { or equivalent }\end{array}$ & 0.55 & $0.34,0.89$ & 0.016 \\
\hline \multirow[t]{3}{*}{ Sought oral health care } & $\begin{array}{l}\text { Frequent need for } \\
\text { dental care }\end{array}$ & 0.58 & $0.35,0.98$ & 0.043 \\
\hline & Medi-Cal insurance & 1.95 & $1.14,3.34$ & 0.015 \\
\hline & No health insurance & 0.50 & $0.30,0.83$ & 0.008 \\
\hline
\end{tabular}


$p=0.295)$. The SF-12 PCS was not significantly associated with needing frequent oral health care $(\mathrm{OR}=0.98 ; 95 \%$ CI $0.96,1.00)$. Once high school education was entered into a multivariate model, no other variables were statistically significant.

\section{Seeking Dental Care}

Participants with Medi-Cal had greater odds of seeking dental care than those lacking Medi-Cal in the bivariate analysis $(\mathrm{OR}=1.95,95 \% \mathrm{CI} 1.14,3.34 ; p=0.015)$. Participants without insurance had $50 \%$ lower odds of seeking dental care $(\mathrm{OR}=$ $0.50,95 \%$ CI $0.30,0.83 ; p=0.008)$. Medi-Cal and no insurance are collinear variables as there were not many participants with other forms of insurance. Participants who reported a frequent need for dental care (six times in 6 months) had a lower odds of seeking dental care than participants who did not report a frequent need for dental care $(\mathrm{OR}=0.58 ; 95 \%$ CI $0.35,0.98 ; p=0.043)$. The SF-12 PCS was not associated with seeking oral health care $(\mathrm{OR}=1.00 ; 95 \%$ CI 0.99 , 1.03). In the multivariate model, only not having insurance $(\mathrm{AOR}=0.53 ; 95 \% \mathrm{CI}$ $0.33,0.85 ; p=0.008)$ and needing frequent dental care $(\mathrm{AOR}=0.57 ; 95 \% \mathrm{CI} 0.35$, $0.93 ; p=0.024)$ were significant.

\section{DISCUSSION}

This paper provides evidence that homeless IDUs in San Francisco have a substantial unmet need for physical and oral health care services. High prevalence of health care needs among IDUs has been documented elsewhere. In Miami, Florida, Chitwood et al. found that $89 \%$ of IDUs reported a need for health care in the past 12 months, consistent with our finding that $82 \%$ of participants reported a need for physical care in the past 6 months. ${ }^{18}$ In our sample, $64 \%$ of participants reported a need for oral health care in the past 6 months, which is higher than a population of IDUs studied by Metsch et al. also in Miami (47\% in the previous year). ${ }^{16}$ This higher prevalence of need in San Francisco may be in part because our whole sample of IDUs was homeless.

The low mean SF-12 Physical Component Score from our population is suggestive of the generally poor health status of homeless IDUs as compared to similarly aged and housed non-IDUs. Burdine et al. have documented that the SF-12 physical and mental health summary scores can serve as a useful measure of community health status when compared with more traditional means of measuring health status, for example through age-adjusted mortality rates or physician to population ratios. $^{26}$ In this analysis, the SF-12 Physical Component Score was associated with health needs and care-seeking behavior.

The use of prescription opiates and benzodiazepines was associated with increased odds of needing frequent health care. Although our cross-sectional study design cannot evaluate causality, we hypothesize that participants with the greatest health needs may have sought prescription medications from healthcare providers. Alternatively, participants could have procured prescription opiates and benzodiazepines through unofficial channels in order to self-medicate for chronic pain, insomnia, or anxiety. Prescription opiate and benzodiazepine use were collinear with methadone treatment, suggesting the use of benzodiazepines in methadone treatment programs.

We found that many homeless IDUs reported needing frequent oral health care, and that having Medi-Cal insurance was significantly associated with increased odds 
of seeking oral health care. In July of 2009, the California State Assembly voted to cut the state's adult dental coverage for Medi-Cal for an estimated savings of 109 million USD. ${ }^{29}$ However, in a report issued by the Oral Health Access Council, the authors argue that the short-term savings would likely evaporate quickly due to a loss of matching federal funds and increased emergency costs. ${ }^{30}$ Cuts to state Medicaid dental benefits have been associated with increased visits to emergency departments in previous studies. ${ }^{31}$ For example, when Maryland legislators eliminated Medicaid reimbursement of dentists for treatment of adults with dental emergencies in 1993, the University of Maryland's emergency department experienced a $22 \%$ increase in dental visits by Medicaid clients. ${ }^{31}$ A reduction in preventive oral health care through the elimination of the statewide Medi-Cal dental program may lead to greater demands for acute dental care and an increased burden on emergency departments.

Participants had injected drugs for an average of 22 years. Bourgois has described the life circumstances of injection drug users as chaotic, interwoven with poverty, homelessness, violence, and addiction. ${ }^{32}$ Under these conditions where basic needs for food and shelter go unmet, injection drug users often wait until a crisis situation in order to seek oral health care. ${ }^{16}$ Moreover, heroin is a potent analgesic which may mitigate pain from all but the most severe oral health problems. ${ }^{10}$

HIV serostatus was not associated with the four outcome variables. Although some have estimated that almost all patients with HIV infection will contract oral diseases, ${ }^{33}$ we found no statistically significant associations between HIV serostatus and oral health. In our study of homeless injection drug users, decades of substance use, poverty, homelessness, and chronic health problems may predispose the entire population to poor oral health. While HIV may contribute to poor oral health, it is not independently statistically associated with greater oral health needs or careseeking behavior. Furthermore, oral health problems may manifest in different stages of HIV infection; for example, oral candidiasis has been found to affect approximately one third of HIV-seropositive patients and more than $90 \%$ of patients with AIDS. ${ }^{34}$ We measured HIV seroprevalence via oral fluid testing but did not collect the laboratory data that would be necessary to assess disease progression.

Our study did not find a statistically significant association between methamphetamine use (injected or non-injected) and a frequent need for dental care. The concept of "Meth Mouth" has been popularized in the media. It refers to a belief that people who use methamphetamine suffer severe consequences in terms of poor oral health. In addition to poor lifestyle and hygiene practices of methamphetamine users, the drug has been hypothesized to contribute to reduced salivation (xerostomia), leading users to drink sugared sodas, which further exacerbates the risk for caries associated with salivary hypofunction. ${ }^{12-14}$ Persistent teeth-grinding (bruxism) and clenching have also been hypothesized to be more common among methamphetamine users and has been informally associated with tooth loss. The link between methamphetamine use and xerostomia has been questioned. ${ }^{13}$ Methamphetamine per se may not be responsible for poor oral health, as the effects of poverty, homelessness, and poor personal hygiene may have similar effects.

In bivariate analysis, we found that methadone treatment (maintenance or detoxification) was associated with increased odds of having sought health care. Increased rates of health care-seeking behavior among participants who had been on methadone treatment may be due to addiction treatment programs having on-site medical care or referring to such care. The lack of any bivariate association between methadone use and seeking oral care is suggestive of methadone maintenance and 
detoxification programs not providing the same level of linkages to oral health services as they do to non-dental medical care. Methadone treatment programs have been identified as a convenient and cost-effective venue for providing supplementary health and psychiatric services. ${ }^{35}$ Given the recent elimination of California's adult dental coverage, methadone treatment centers could serve as important venues for non-emergency dental care.

Participants who reported a frequent need for dental care had lower odds of seeking oral health care than participants who did not report a frequent need for dental care. This counterintuitive finding may indicate that some participants with oral health problems may have sought care in the past and had their concerns addressed, while those who did not seek dental care continued to experience oral health problems. In other words, rather than oral health need predicting careseeking behavior, care-seeking behavior predicted frequent oral health needs.

This study has several limitations that should be considered when interpreting its results. Participants were recruited using two distinct methodologies, targeted sampling in two neighborhoods and convenience sampling in another neighborhood. It is possible that the group recruited through convenience sampling was qualitatively different than the samples recruited through targeted sampling. However, our comparisons found few differences attributable to neighborhood or sampling design, and we controlled for these variables in analyses. In the analysis, health status was measured using the Short Form 12 Physical Component Score, which is a self-reported measure rather than a clinical assessment. The study lacked a validated oral health instrument, such as the Oral Health Impact Profile, which is a scaled index of the social impact of oral disorders. ${ }^{36}$ The primary outcomes in the study, the self-reported need for health and dental care, are subjective measures that could vary widely by individual. The study would have been much improved by clinical observations of physical and oral health, and expert opinion about the need for health or dental care. In future studies the authors hope to measure the number of decayed, missing, and filled teeth, a standard index that has been applied to homeless populations. ${ }^{37}$ A majority of participants reported seeking care "elsewhere." In our study questionnaire we did not capture the specific sources of physical and oral health care, something that would be important to do in future studies. Study participants were asked about their care-seeking behaviors, but they were not asked if they received care. It would be misleading to assume that treatment-seeking resulted in treatment in every case. In future studies participants should be asked more detailed questions about the receipt of medical or dental treatment, including reasons why care was sought but not received. Further research should also explore the feasibility and acceptability of various oral health care preventive strategies. Finally, tobacco usage was not assessed, which is a potentially important confounding variable that should be accounted for in future studies.

\section{CONCLUSION}

In San Francisco, homeless IDUs comprise a poly-substance using population with frequent needs for physical and oral health care. Predictably, the health status of this population was measured to be significantly lower than the general population. Medi-Cal coverage and a history of methadone treatment were statistically significant correlates of health and oral health care-seeking behavior. Rather than cutting access to care among this vulnerable population, it may be more effective public health policy to increase preventive and treatment services. 


\section{ACKNOWLEDGMENT}

This study was funded by NIDA grant R01 DA016019 (Elizabeth Lambert, Program Officer). We want to thank the research staff and all of the participants in the project. We would also like to thank the University of California, San Francisco's Dean's Summer Fellowship for medical students for financial support.

Financial Disclosures. None of the authors report any financial relationships or other conflicts of interest.

OPEN ACCESS This article is distributed under the terms of the Creative Commons Attribution Noncommercial License which permits any noncommercial use, distribution, and reproduction in any medium, provided the original author(s) and source are credited.

\section{REFERENCES}

1. Neale J. Making the absent body present. Addiction. 2008; 103(11): 1821-1825.

2. Garfein RS, Vlahov D, Galai N, Doherty MC, Nelson KE. Viral infections in short-term injection drug users: the prevalence of the hepatitis $\mathrm{C}$, hepatitis $\mathrm{B}$, human immunodeficiency, and human T-lymphotropic viruses. Am J Public Health. 1996; 86(5): 655-661.

3. Deiss RG, Rodwell TC, Garfein RS. Tuberculosis and illicit drug use: review and update. Clin Infect Dis. 2009; 48(1): 72-82.

4. Tempalski B, Lieb S, Cleland CM, Cooper H, Brady JE, Friedman SR. HIV prevalence rates among injection drug users in 96 large US metropolitan areas, 1992-2002. J Urban Health. 2009; 86(1): 132-154.

5. Sporer KA, Kral AH. Prescription naloxone: a novel approach to heroin overdose prevention. Ann Emerg Med. 2007; 49(2): 172-177.

6. Reece AS. Dentition of addiction in Queensland: poor dental status and major contributing drugs. Aust Dent J. 2007; 52(2): 144-149.

7. Laslett AM, Dietze P, Dwyer R. The oral health of street-recruited injecting drug users: prevalence and correlates of problems. Addiction. 2008; 103(11): 1821-1825.

8. Reece AS. An intriguing association between dental and mental pathology in addicted and control subjects: a cross-sectional survey. Br Dent J. 2008; 205(11): E22.

9. Reece S. Dental health in addiction. Aust Dent J. 2009; 54(2): 185-186.

10. Rosenstein DI. Effect of long-term addiction to heroin on oral tissues. $J$ Public Health Dent. 1975; 35(2): 118-122.

11. Colon PG. Heroin abuse and its effects on teeth. Quintessence Int Dent Dig. 1975; 6(10): 103-108.

12. Klasser GD, Epstein J. Methamphetamine and its impact on dental care. J Can Dent Assoc. 2005; 71(10): 759-762.

13. Shaner JW, Kimmes N, Saini T, Edwards P. "Meth mouth": rampant caries in methamphetamine abusers. AIDS Patient Care STDS. 2006; 20(3): 146-150.

14. Padilla R, Ritter AV. Meth mouth: methamphetamine and oral health. J Esthet Restor Dent. 2008; 20(2): 148-149.

15. Shetty V, Mooney LJ, Zigler CM, Belin TR, Murphy D, Rawson R. The relationship between methamphetamine use and increased dental disease. J Am Dent Assoc. 2010; 141(3): 307318.

16. Metsch LR, Crandall L, Wohler-Torres B, Miles CC, Chitwood DD, McCoy CB. Met and unmet need for dental services among active drug users in Miami, Florida. J Behav Health Serv Res. 2002; 29(2): 176-188.

17. Fan J, Hser YI, Herbeck D. Tooth retention, tooth loss and use of dental care among long-term narcotics abusers. Subst Abus. 2006; 27(1-2): 25-32. 
18. Chitwood DD, McBride DC, French MT, Comerford M. Health care need and utilization: a preliminary comparison of injection drug users, other illicit drug users, and nonusers. Subst Use Misuse. 1999; 34(4-5): 727-746.

19. Chitwood DD, Sanchez J, Comerford M, McCoy CB. Primary preventive health care among injection drug users, other sustained drug users, and non-users. Subst Use Misuse. 2001; 36(607): 807-824.

20. Chitwood DD, Comerford M, McCoy HV. Satisfaction with access to health care among injection drug users, other drug users, and nonusers. J Behav Health Serv Res. 2002; 29 (2): 189-197.

21. Heinzerling KG, Kral AH, Flynn NM, et al. Unmet need for recommended preventive health services among clients of California syringe exchange programs: implications for quality improvement. Drug Alcohol Depend. 2006; 81(2): 167-178.

22. French MT, McGeary KA, Chitwood DD, McCoy CB. Chronic illicit drug use, health services utilization and the cost of medical care. Soc Sci Med. 2000; 50(12): 1703-1713.

23. McGeary KA, French MT. Illicit drug use and emergency room utilization. Health Serv Res. 2000; 35(1 Pt 1): 153-169.

24. Watters JK, Biernacki P. Targeted sampling: options for the study of hidden populations. Social Problems. 1989; 36(4): 416-430.

25. Kral AH, Bluthenthal RN, Lorvick J, Gee L, Bacchetti P, Edlin BR. Sexual transmission of HIV-1 among injection drug users in San Francisco, USA: risk-factor analysis. Lancet. 2001; 357(9266): 1397-1401.

26. Burdine JN, Felix MR, Abel AL, Wiltraut CJ, Musselman YJ. The SF-12 as a population health measure: an exploratory examination of potential for application. Health Serv Res. 2000; 35(4): 885-904.

27. Riley ED, Bangsberg DR, Perry S, Clark RA, Moss AR, Wu AW. Reliability and validity of the SF-36 in HIV-infected homeless and marginally housed individuals. Qual Life Res. 2003; 12(8): 1051-1058.

28. StataCorp. Stata Statistical Software: Release 10. College Station: StataCorp LP; 2007.

29. Colliver V. Loss of Medi-Cal dental care hard to swallow. San Francisco Chronicle. 2009.

30. Hughes, Diringer. Eliminating Medi-Cal adult dental: Costs and consequences. Oral Health Access Council. 2009.

31. Cohen LA, Manski RJ, Hooper FJ. Does the elimination of Medicaid reimbursement affect the frequency of emergency department dental visits? J Am Dent Assoc. 1996; 127 (5): 605-609.

32. Bourgois P. The moral economies of homeless heroin addicts: confronting ethnography, HIV risk, and everyday violence in San Francisco shooting encampments. Subst Use Misuse. 1998; 33(11): 2323-2351.

33. Weinert M, Grimes RM, Lynch DP. Oral manifestations of HIV infection. Ann Intern Med. 1996; 125(6): 485-496.

34. McCarthy GM. Host factors associated with HIV-related oral candidiasis. A review. Oral Surg Oral Med Oral Pathol. 1992; 73(2): 181-186.

35. Umbricht-Schneiter A, Ginn DH, Pabst KM, Bigelow GE. Providing medical care to methadone clinic patients: referral vs on-site care. Am J Public Health. 1994; 84(2): 207210.

36. Slade GD, Spencer AJ. Development and evaluation of the oral health impact profile. Community Dent Health. 1994; 11(1): 3-11.

37. Conte M, Broder HL, Jenkins G, Reed R, Janal MN. Oral health, related behaviors and oral health impacts among homeless adults. J Public Health Dent. 2006; 66(4): 276-278. 